Eur J Surg Oncol 2019 May 16. pii: S0748-7983(19)30454-8. (doi: 10.1016/j.ejso.2019.05.016.) Open Access Green (Postprint version)

Link to Publisher version:

https://www.sciencedirect.com/science/article/pii/S0748798319304548?via\%3Dihub

\title{
Sentinel lymph node biopsy following previous axillary surgery in recurrent breast cancer
}

\section{Á. Sávolt ${ }^{1}{ }^{*}$, G. Csernii $^{2,3}$, Gy. Lázár ${ }^{4}$, R. Maráz ${ }^{5}$, P. Kelemen $^{1}$, E. Kovács ${ }^{6}$, B. Győrffy ${ }^{7}$, N. Udvarhelyi ${ }^{8}$, A. Vörös ${ }^{3}$, K. Ormandi $^{9}$, Z. Mátrai ${ }^{1}$}

${ }^{1}$ Department of Breast and Sarcoma Surgery, National Institute of Oncology, Budapest, Hungary

${ }^{2}$ Department of Pathology, Bács-Kiskun County Teaching Hospital, Kecskemét, Hungary

${ }^{3}$ Department of Pathology, University of Szeged, Szeged, Hungary

${ }^{4}$ Department of Surgery, University of Szeged, Szeged, Hungary

${ }^{5}$ Department of Surgery and Oncology, Bács-Kiskun County Teaching Hospital, Kecskemét, Hungary

${ }^{6}$ Department of Diagnostic Imaging, National Institute of Oncology, Budapest, Hungary

${ }^{7}$ MTA TTK Momentum Cancer Biomarker Res. Group, Hungarian Academy of Sciences, Budapest, Hungary

${ }^{8}$ Department of Diagnostic Imaging, National Institute of Oncology, Budapest, Hungary

${ }^{9}$ Affidea Diagnostics, Mammography Unit, Szeged, Hungary

* Corresponding author. Department of Breast and Sarcoma Surgery, National Institute of Oncology, H-1122, Rath Gy u. 7.-9., Budapest, Hungary Tel.: +36-1-2248600/3444

E-mail address: drsavolt@hotmail.com(Á. Sávolt). 


\begin{abstract}
Introduction

Ipsilateral breast recurrence or second primary breast cancer can develop in patients who have undergone breast conserving surgery (BCS) and axillary surgery. The purpose of this study was to examine the feasibility of a reoperative sentinel lymph node biopsy (SLNB) as a repeated axillary staging procedure.
\end{abstract}

\title{
Patients and Methods
}

From August 2014 through January 2017 patients with locally recurrent breast cancer or with BRCA mutation requiring risk reduction mastectomy as a second surgical procedure, underwent repeat SLNB in three Hungarian Breast Units with a radiocolloid (and blue dye) technique.

\section{Results}

Hundred and sixty repeat SLNBs were analysed, 80 after previous SLNB and 80 after previous total or partial axillary lymph node dissection (ALND). SLN identification was successful in 106 patients (66\%); 62/80 (77.5\%) and 44/80 (55\%) in the SLNB and ALND groups, respectively. $(\mathrm{p}<0.003)$. Extra-axillary lymph drainage was more frequent in the ALND group (19/44, 43,2\% versus 7/62, 11,3\%;p<0.001). Lymphatic drainage to the contralateral axilla was observed in 14 patients ( 11 in the ALND group, $\mathrm{p}=0.025$ ), isolated parasternal drainage was detected in 4 patients $(\mathrm{p}=0.31$ ). Only $9 / 106$ patients with successful repeat SLNB $(8,8 \%$, all with 1 SLN removed) had SLN metastases

Repeat SLNB is feasible and accurate-for $66 \%$ of in patients with ipsilateral breast tumor recurrence or new ipsilateral primary tumor after previous BCS and axillary staging,

especially after a previous SLNB. Repeat SLNB should replace routine ALND as the standard axillary restaging procedure in recurrent disease with a clinically negative axilla. Preoperative lymphoscintigraphy is important to explore extra-axillary lymphatic drainage in this restaging setting. 


\section{Introduction}

For all breast cancers, the local recurrence rate following breast conserving surgery (BCS) is reported to be about $5-10 \%$ at ten years follow-up (1-3). There is no definite consensus regarding the best strategy for managing the regional lymph nodes in patients with local recurrences and clinically negative axilla $(4-5,23)$. Similarly, there has been no prospective, randomized trial that showed a survival or regional control benefit of completion axillary lymph node dissection (ALND) for patients with local recurrence after BCS with sentinel lymph node biopsy (SLNB) (6). Previous breast cancer treatment with surgery and/or radiotherapy of the breast and axilla could lead to disrupted lymph drainage by scar tissue and fibrosis (7). A previously operated axilla was considered to be a relative contraindication for performing an SLNB, but there is evidence supporting the use of SLNB in the reoperative setting (8-10). Lymphatic drainage after previous breast surgery would be altered and it remains questionable whether SLNB at the time of surgery for locally recurrent breast cancer (repeat SLNB, reSLNB) is technically feasible, and whether ALND can safely be omitted if repeat SLNB yields lymph nodes free of metastasis (11-14). The aim of this study is to evaluate technical feasibility of performing reoperative SLNB in patients with ipsilateral recurrent breast cancer.

\section{Patients and Methods}

From, patients with locally recurrent breast cancer or BRCA gene germline mutation carriers in need of risk reduction mastectomy as a second surgical procedure who underwent re-SLNB in three Hungarian Breast Units from August 2014 through January 2017 formed the study population.

\section{SLNB procedure and axillary management}

Lymphatic mapping was performed using technetium-99m-labeled tin colloid (particle diameter: $0.2-0.4 \mathrm{~mm}$ ) or albumin (particle size up to $0.1 \mu \mathrm{m}$ ). The colloid was injected periareolary or peritumorally on the day before the operation. Lymphoscintigraphy was performed on the next morning. In case of contralateral axillary drainage, the removal of the 
labelled SLNs was attempted, but parasternal (internal mammary) SLNs were not removed. If the lymphoscinfigraphy showed only internal mammary chain drainage, the re-SLNB was unsuccessful, but was recorded as aberrant. Sentinel lymph nodes (SLNs) were identified by a handheld gamma probe (Europrobe, ... France ). The blue dye technique was used either regularly or in patients in whom lymphoscintigraphy failed to highlight a lymph node: $2 \mathrm{ml}$ Patent V blue dye was injected periareolarly or peritumorally. If no axillary SLN could be identified with the methods above, an ALND (after SLNB) or axillary revision (after ALND) was performed depending on the type of axillary surgery performed before. A completion ALND to evaluate the false negative rate of re-SLNB was not routinely performed in patients with successfully identified SLNs. SLNs were fixed in $10 \%$-formalin solution and embedded in paraffin. SLNs greater than $4 \mathrm{~mm}$ were cut into approximately $2 \mathrm{~mm}$ thick slices and step sectiond at 200 or $250 \mu \mathrm{m}$. The least exhaustive histology protocol investigated 3 step sections from each slice with hematoxylin and eosin $(\mathrm{H} \& \mathrm{E})$ staining, whereas the most exhaustive one looked at 5 step sections, 4 consecutive ones with $\mathrm{H} \& \mathrm{E}$, and the fifth with cytokeratin immunohistochemistry, if the former sections were negative.

\section{Statistical analysis}

Patient data were captured on standardized registration forms. Lymphoscintigraphies showing drainage outside the ipsilateral axilla were recorded as aberrant drainage. Descriptive statistics were used in this study. Statistical differences between groups, re-SLNB identification rates and aberrant drainage were determined using the Chi square test. A p value under 0.05 was considered statistically significant. Data were analysed using IBM SPSS Statistics version 19 or 22 (SPSS Inc., Chicago, IL, USA).

\section{Results}

A total of 160 patients were collected from the 3 centres. Of these, 134 were treated at the National Institute of Oncology (NIO) in Budapest, 15 at the Bács-Kiskun County Teaching Hospital in Kecskemét and 11 at the University of Szeged. Fifteen patients with BRCA gene mutations without any recognizable recurrent tumour - all from the NIO - underwent risk reduction mastectomy as a second surgery.

The median age at diagnosis of the recurrence or at the second surgical procedure in case of 
risk reduction oncoplastic mastectomy was 62 years (range 31-87 years). All patients underwent BCS for their primary tumours. Patients were divided into two groups on the basis of previous axillary surgery: 80/160 had SLNB and 80/160 patients had complete or partial (incomplete, with less than 10 axillary lymph nodes removed) ALND before re-SLNB. The mean number of lymph nodes removed at the time of primary axillary surgery with SLNB and ALND was 1.7 (range 1-3) and 13.2 (range 6-21), respectively. Breast irradiation after the primary tumour was performed in 152 patients (95\%) (TABLE1). At the time of second surgery and re-SLNB, oncoplastic mastectomy $(n=28)$, radical mastectomy $(n=105)$ and repeat BCS $(n=27)$ were performed.

\section{Identification rate and aberrant lymph drainage}

Reoperative sentinel node identification was successful in 106 of the 160 patients (66\%). The identification rate was $77.5 \%$ (62/80) in the SLNB group and 55\% (44/80) in the ALND group $(\mathrm{p}=0.003)$. The average number of SLNs removed was 1.65 (range 1-6) overall, 1.63 (range 15) after previous SLNB and 1.68 (range 1-6) after ALND, respectively. Twenty-six patients $(26 / 106,24.5 \%)$ showed aberrant lymph drainage pathways, which was more frequent in the ALND group $(19 / 44,43.2 \%)$ than in the SLNB group $(7 / 62,11.3 \%)(\mathrm{p}<0.001)$ (TABLE2). Lymphatic drainage to the contralateral axilla was observed in 14 patients ( 3 vs. 11 , in the SLNB and the ALND group, respectively $(\mathrm{p}=0.025)$, isolated parasternal drainage was detected in 4 patients $(\mathrm{p}=0.31)$. Sites and numbers of patients with aberrant drainage at second lymphoscintigraphy are shown in TABLE3. There was no patient in whom an SLN could be identified with the blue dye technique, after a failure of lymphoscintigraphy to highlight a lymph node.

Of the 106 patients with successful re-SLNB, 93 (91.2\%) were pathologically nodenegative (rpN0). In 9 (8.8\%) patients, re-SLNBs yielded a metastatic lymph node; all these patients had only 1 SLN removed. In 6 of the 9 involved SLNs only micrometastases (rpNmi) were detected, and no completion ALND was done. In the remaining three patients (rpN1), who all received whole breast irradiation at the time of their first operation, completion ALND was performed as a second procedure. Of the metastases, seven were located in the ipsilateral axilla and two in the contralateral axilla (TABLE4). 


\section{Discussion}

Re-SLNB after previous SLNB, in patients with recurrent breast cancer, second primary breast cancer or prophylactic mastectomy, was successful in $77.5 \%$ of our patient population, which is comparable with the success rates of other studies (12-16). Reoperative SLNB failed to detect new SLNs in 18 of 80 patients (22.5\%). We tried to performe reSLNB in patients after previous total or partial (incomplete) ALND as well. Sentinel lymph node identification was successful in 44 of 80 patients $(55 \%)$, but aberrant lymphatic drainage was more frequent $(19 / 44,43.2 \%)$ in this setting.

Previous breast or axillary surgery can partially or temporarily interrupt and modify lymphatic flow. The main argument against re-SLNB is that the lymphatic channels and drainage are considered to be disrupted due to fibrosis after axillary surgery and radiotherapy (17-19). Therefore, it is important to use preoperative lymphoscintigraphy to explore whether new SLNs can be identified. The reported percentage of "aberrant" lympatic drainage pathways outside the ipsilateral axilla in patients with previous BCS and axillary surgery is $2-47 \%$ (2021)._Ploeg suggested that the drainage outside the axilla in the treated breast is higher than that in the untreated breast (51 vs. $33 \%, \mathrm{P}=0.003$ ) (22). Schrenk et al. demonstrated a significant correlation between the identification rate and a positive lymphoscintigraphy, therefore, they recommend performing lymphoscintigraphy at the time of surgery for ipsilateral breast cancer recurrence (13). They also underline the usefulness of indo-cyanine green (ICG) fluorescence method for identifying aberrant lymphatic drainage.

Maaskant-Braat et al, also reported that aberrant drainage was seen more frequently in cases after previous ALND than in those with previous SLNB (79.3 vs. $25.0 \%, \mathrm{P}=0.0001)$ (10). A recent systematic review and meta-analysis by Maaskant-Braat et al. analysed 692 from 25 studies patients with locally recurrent breast cancer who underwent re-SLNB. Of these patients, 301 had undergone previous SLNB, 361 had undergone ALND. The overall identification rate was $65.3 \%$ (452 of 692 patients), but the rate was significantly higher for patients who had undergone previous SLNB (81.0\%, 243 of 301 patients) than for patients who had undergone ALND (52.2\%, 166 of 318 patients). Aberrant drainage pathways were visualized in $43.2 \%$ 
of these patients, which was more frequent after ALND than after SLNB (69\% vs 17\% among patients with successful lymphatic mapping, and 33\% vs 14\% among all patients). Although completion ALND was not done in all patients with negative SLN, there were no falsenegatives among 63 SLN-negative procedures validated by a planned ALND, and subsequent axillary recurrence developed in only one of these latter patients (8).

The concept of repeat SLNB has emerged in recent years, but still no consensus on guidelines exists regarding the optimal management of lymph nodes in patients with ipsilateral breast cancer recurrence and the impact of nodal involvement on prognosis is still unclear. It is possible, that the positive re-SLNB in recurrent breast cancer does not have the same importance in the choice of adjuvant systemic therapy as in the primary breast cancer (24). The CALOR (chemotherapy for isolated locoregional breast cancer) trial found, that adjuvant chemotherapy should be recommended for patients with completely resected isolated locoregional recurrences of breast cancer, especially if the recurrence is oestrogen receptor negative (25). Although systemic therapy given to locoregional recurrence improved survival in this trial, removal of the locoregional relapse was also mandated in this trial, and re-SLNB may also help in tailoring local therapy, when only SLNB had been done previously. Ugras et al. also reported that the re-SLNB is worthwhile in all patients with invasive local recurrences and clinically negative nodes, but the use of systemic therapy and subsequent radiation is increasingly defined by the tumor subtype rather than by nodal involvement (26).

\section{Conclusions}

$\underline{\text { Our findings are consistent with prior studies which imply that re-SLNB is feasible, and }}$ may provide a conservative alternative to ALND for breast cancer patients (whether with a new primary or a recurring cancer) who have had a previous axillary operation, SLNB or sometimes (partial) ALND. We propose that re-SLNB is a further step in deescalating radical axillary surgery in the circumstances described above, but its value in treatment tailoring, its morbidity and its comparability to no further surgery has to be established. The current axillary managment in recurrent breast cancer is an additional axillary evaluation with re-SLNB for all patients who are clinically node negative (including axillary ultrasound evaluation in the assessment) following BCT and SLNB. We recommend to use preoperative lymphoscintigraphy, paying attention to the contralateral axilla and the internal mammary 
nodes. As the finding of locoregional recurrence or new primary breast cancer generally implies adjuvant systemic therapy, the results of nodal involvement are rarely expected to change recommendations on adjuvant systemic therapy; however they may change indications of radiotherapy (for non irradiated regions). In such cases contralateral and internal mammary nodal excision may be indicated.

\section{Acknowledgment}

This study was partially funded by the National Research, Development and Innovation Office grant GINOP-2.3.2-15-2016-00020. 


\section{References}

1. Early Breast Cancer Trialists' Collaborative Group (EBCTCG), Darby S, McGale P, Correa C, Taylor C, Arriagada R, Clarke M, et al. Effect of radiotherapy after breastconserving surgery on 10-year recurrence and 15-year breast cancer death: metaanalysis of individual patient data for 10,801 women in 17 randomised trials. Lancet. 2011;378:1707-16.

2. Wapnir IL, Anderson SJ, Mamounas EP, Geyer CE Jr, Jeong JH, Tan-Chiu E et al (2006) Prognosis after ipsilateral breast tumor recurrence and locoregional recurrences in five National Surgical Adjuvant Breast and Bowel Project node-positive adjuvant breast cancer trials. J Clin Oncol 24(13):2028-2037

3. Cossetti RJ, Tyldesley SK, Speers CH, Zheng Y, Gelmon KA.Comparison of breast cancer recurrence and outcome patterns between patients treated from 1986 to 1992 and from 2004 to 2008. J Clin Oncol. 2015 Jan 1;33(1):65-73.

4. Derkx F, Maaskant-Braat AJ, van der Sangen MJ, Nieuwenhuijzen G.A, van de PollFranse, LV, Roumen RM et al. Staging and management of axillary lymph nodes in patients with local recurrence in the breast or chest wall after a previous negative sentinel node procedure. Eur J Surg Oncol. 2010 Jul; 36(7):646-51.

5. Tasevski R, Gogos AJ, Mann GB: Reoperative sentinel lymph node biopsy in ipsilateral breast cancer releapse. The Breast 2009: 18: 322-326.

6. Saji S, Hiraoka M, Tokuda Y, Fukui N, Ikeda T. Trends in local therapy application for early breast cancer patients in the Japanese Breast Cancer Society Breast Cancer Registry during 2004-2009. Breast Cancer. 2012;19:1-3.

7. Tada K, Nishimura S, Miyagi Y, Takahashi K, Makita M, Iwase T, et al. The effect of an old surgical scar on sentinel node mapping in patients with breast cancer: a report of five cases. Eur J Surg Oncol. 2005;31:840-4.

8. Maaskant-Braat AJG, Voogd AC, Roumen RMH, Nieuwehuijzen GAP: Repeat sentinel node biopsy in patients with locally recurrent breast cancer: a systematic review and meta- analysis of the literature. Breast Cancer Res Treat 2013: 138:13-20.

9. Intra M, Trifirò G, Galimberti V, Gentilini O, Rotmensz N, Veronesi P. Second axillary sentinel node biopsy for ipsilateral breast tumour recurrence. Br J Surg. 2007 Oct;94(10):1216-9.

10. Maaskant-Braat AJ, Roumen RM, Voogd AC, Pijpers R, Luiten EJ, Rutgers EJ, et al. Sentinel Node and Recurrent Breast Cancer (SNARB): results of a nationwide registration study. Ann Surg Oncol. 2013;20:620-6.

11. Kothari MS, Rusby JE, Agusti AA, MacNeill FA.Sentinel lymph node biopsy after previous axillary surgery: A review. Eur J Surg Oncol. 2012 Jan;38(1):8-15. doi: 10.1016/j.ejso.2011.10.003. Epub 2011 Oct 26.

12. Kaur P, Kiluk JV, Meade T, Ramos D, Koeppel W, Jara J, et al. Sentinel lymph node biopsy in patients with previous ipsilateral complete axillary lymph node dissection. Ann Surg Oncol. 2011;18:727-32.

13. Schrenk P, Tausch C, Wayand W. Lymphatic mapping in patients with primary or recurrent breast cancer following previous axillary surgery. Eur J Surg Oncol. 2008;34:851-6.

14. Cox CE, Furman BT, Kiluk JV, Jara J, Koeppel W, et al. Use of reoperative sentinel lymph node biopsy in breast cancer patients. J Am Coll Surg. 2008;207(1):57-61.

15. Port ER, Garcia-Etienne CA, Park J, et al. Reoperative sentinel lymph node biopsy: a new frontier in the management of ipsilateral breast tumor recurrence. Ann Surg Oncol 2007;14: 2209-2214. 
16. Barone JL, Feldman SM, Estabrook A, Tartter PI, Rosenbaum Smith SM, Boolbol SK. Reoperative sentinel lymph node biopsy in patients with locally recurrent breast cancer. Am J Surg. 2007;194:491-3.

17. Agarwal A, Heron DE, Sumkin J, Falk J. Contralateral uptake and metastases in sentinel lymph node mapping for recurrent breast cancer. J Surg Oncol 2005; 92.4-8.

18. Sood A, Youssef IM, Heiba SI, El-Zeftawy H, Axelrod D, Seigel B et al. Alternative lymphatic pathway after previous axillary node dissection in recurrent/primary breast cancer. Clin Nucl Med 2004; 29: 698-702.

19. Newman EA, Cimmino VM, Sabel MS, Diehl KM, Frey KA, Chang AE et al. Lymphatic mapping and sentinel lymph node biopsy for patients with local recurrence after breastconservation therapy. Ann Surg Oncol 2006; 13: 52-57.

20. Luini A, Galimberti V, Gatti G, Arnone P, Vento AR, Trifiro G et al. The sentinel node biopsy after previous breast surgery: preliminary results on 543 patients treated at the European Institute of Oncology. Breast Cancer Res Treat 2005; 89: 159-163.

21. Yamashita T, Fujita T, Hayashi H, Ando Y, Hato Y, Horio A, et al. Detection of parasternal metastatic lymph nodes by sentinel lymph node methods in a patient with recurrence in the conserved breast. Breast Cancer. 2011.

22. Van der Ploeg IM, Oldenburg HS, Rutgers EJ, Baas-Vrancken Peeters MJ, Kroon BB, et al. Lymphatic drainage patterns from the treated breast. Ann Surg Oncol. 2010;17:1069-75.

23. Savolt A, Peley G, Polgar C, Udvarhelyi N, Rubovszky G, Kovacs E, Gyorffy B, Kasler M, Matrai Z. Eight-year follow up result of the OTOASOR trial: The Optimal Treatment Of the Axilla - Surgery Or Radiotherapy after positive sentinel lymph node biopsy in early-stage breast cancer: A randomized, single centre, phase III, non-inferiority trial.Eur J Surg Oncol 2017, 43, 672-9.

24. Poodt, Ingrid GM, et al. "Repeat sentinel lymph node biopsy for ipsilateral breast tumor recurrence: a systematic review of the results and impact on prognosis." Annals of surgical oncology(2018): 1-11.

25. Wapnir, Irene L., et al. "Efficacy of chemotherapy for ER-negative and ER-positive isolated locoregional recurrence of breast cancer: final analysis of the CALOR trial." Journal of clinical oncology 36.11 (2018): 1073-1079.

26. Ugras, Stacy, et al. "Reoperative sentinel lymph node biopsy is feasible for locally recurrent breast cancer, but is it worthwhile?." Annals of surgical oncology 23.3 (2016): 744-748. 